\title{
¿Por qué los niños y las niñas se aceptan y se rechazan?
}

\author{
M $^{\mathrm{a}}$ INÉS MONJAS ${ }^{1}$, INMACULADA SUREDA ${ }^{2}$ \\ Y FRANCISCO-JUAN GARCÍA-BACETE ${ }^{3 *}$ \\ ${ }^{1}$ Universidad de Valladolid; ${ }^{2}$ Universidad de las Islas Baleares; \\ ${ }^{3}$ Universidad Jaime I de Castellón
}

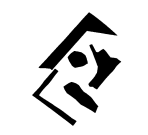

\section{Resumen}

En el presente estudio se pretende elaborar un sistema de categorías de los motivos que los niños utilizan para justificar sus elecciones y rechazos. Con las respuestas emitidas en un cuestionario sociométrico de nominaciones positivas y negativas por 430 alumnos de quinto y sexto curso de Educación Primaria, pertenecientes a 21 aulas de centros públicos de Valladolid, Palma de Mallorca y Castellón de la Plana, se estableció un sistema con nueve categorias de aceptación y quince de rechazo. Las principales razones por las que se elige un amigo son la simpatía, la diversión y satisfacción conjunta y la presencia de características relevantes en una relación de amistad (confianza, lealtad, apoyo, ayuda...). Los principales motivos de rechazo son las conductas agresivas, especialmente la agresión psicológica (dominancia y superioridad, básicamente prepotencia), también la agresión física, la agresión verbal y gestual, y los comportamientos que revelan inmadurez y falta de atención y los que agobian y/o resultan molestos para los otros. Esta taxonomía de aceptaciones y rechazos puede ser la base para desarrollar programas de competencia social.

Palabras clave: Aceptación, rechazo, sociometría, nominación entre iguales, preferencia social.

\section{Why do children accept and reject each other?}

\begin{abstract}
The present study attempts to elaborate a category system of the reasons children give to justify their preferences and rejections. Using the answers given in a sociometric questionnaire consisting on positive and negative nominations by 430 pupils, in the fifth and sixth years of Primary Education, from 21 classes, in state schools in Valladolid, Palma de Mallorca, and Castellón de la Plana (Spain), a system was established with nine acceptance and fifteen rejection categories. The main reasons for choosing a friend include: friendliness, fun, and joint satisfaction, together with the presence of relevant characteristics for a friendship relation (trust, loyalty, support, help...). The main reasons for rejection are: aggressive behaviour, especially psychological aggression (dominance and superiority -basically arrogance), as well as physical, verbal and gestural aggression. Those whose behaviour reveals immaturity and lack of care also tend to be rejected, as do those who overwhelm and/or annoy others. This taxonomy of acceptance and rejection could be the basis for developing social skill programmes.
\end{abstract}

Keywords: Acceptance, peer rejection, sociometry, peer-nomination techniques, social preference.

\footnotetext{
*Miembros del Grupo GREI (Grupo de Investigación Interuniversitario del Rechazo entre Iguales en el Contexto Escolar).

Agradecimientos: Investigación realizada gracias a la ayuda de investigación "El rechazo entre iguales en la vida cotidiana" (SEJ2004-04028) concedida por el Ministerio de Ciencia y Tecnología (Plan Nacional de I+D+I, 2004-07).

Correspondencia con los autores: $\mathrm{M}^{\mathrm{a}}$ Inés Monjas Casares. Departamento de Psicología. Facultad de Educación y Trabajo Social. Universidad de Valladolid. Campus Miguel Delibes. Paseo Belén, 1. 47011 Valladolid. Tel.: 983184354. Fax: 983423436.E-mail: imonjas@psi.uva.es.

Original recibido: Junio, 2007. Aceptado: Febrero, 2008.
} 


\section{Introducción}

Dentro de las relaciones entre iguales un aspecto que ha suscitado interés es la aceptación social, entendida como el grado en que un niño es querido, aceptado, reconocido y apreciado en su grupo de iguales. Ello es consecuencia de la importancia que tiene la atracción interpersonal como índice de ajuste ya que la evidencia indica que la adecuada aceptación en el grupo de iguales está asociada con buena adaptación mientras que la baja aceptación social, la ignorancia y el rechazo son factores de riesgo importantes para la desadaptación (Bukowski, Newcomb y Hartup, 1998; Newcomb, Bukowski y Pattee, 1993). El que un niño sea aceptado significa que los compañeros buscan su compañía y su amistad y que le estiman, le valoran y le quieren, mientras que la ignorancia, y especialmente el rechazo, denotan desde falta de estima, de atracción y de valoración hasta antipatía y desagrado.

Nuestra atención en este estudio se centra en las relaciones entre iguales que se producen en el aula y concretamente en qué razones esgrimen los niños para justificar tanto sus preferencias y aceptación como sus antipatías y rechazo.

El grado de aceptación de un niño se determina, principalmente, a través de estrategias sociométricas, procedimientos de evaluación que permiten obtener información de la atracción interpersonal entre los miembros del grupo y proporcionan una medida del estatus social del niño. Uno de los métodos más utilizados es el de nominación entre iguales que básicamente consiste en que cada alumno emite una o varias nominaciones positivas y negativas de sus compañeros del grupo-clase de acuerdo a un criterio determinado, por ejemplo buenos amigos, compañero de juegos... Esta estrategia de evaluación es ampliamente utilizada en la investigación de la aceptación social en contextos escolares (Bukowski, Sippola, Hoza y Newcomb, 2000; Cava y Musitu, 2001; Foster, Inderbitzen y Nagle, 1999; García-Bacete, 2006; 2007).

De acuerdo con el número de nominaciones positivas y negativas recibidas y siguiendo la tipología de Coie, Dodge y Coppotelli (1982) se contemplan cinco tipos sociométricos: preferidos, rechazados, ignorados, controvertidos y promedios. Los resultados del metaanálisis realizado por Newcomb, Bukowski y Pattee (1993) muestran que los niños de cada tipo sociométrico tienen un repertorio conductual. García-Bacete (2007) ofrece la siguiente caracterización de cada tipo. Los alumnos rechazados son los más agresivos y aislados y los menos sociables. Los preferidos son los más sociables, los menos aislados y sus tasas de agresividad son tan bajas como las de los ignorados. Los ignorados tienen niveles muy bajos de agresividad, bajos de sociabilidad y entre bajo y medio-bajo en aislamiento. Los controvertidos, como los rechazados, siempre tienen tasas por encima de la media en agresividad y aislamiento, pero son menos sociables que los preferidos en la muestra de los pequeños. En trabajos anteriores hemos encontrado los siguientes porcentajes globales para cada tipo sociométrico: 9.9\% preferidos, $11.3 \%$ rechazados, $12.4 \%$ ignorados, $4.2 \%$ controvertidos y $62.1 \%$ promedio (García-Bacete, Sureda y Monjas, 2008).

Además de la nominación directa, en algunos casos se pide a los niños o adolescentes que aporten razones que justifiquen sus elecciones o rechazos, lo cual va a permitir el acercamiento a los motivos que subyacen tanto a las preferencias de relación como a la antipatía y exclusión. Este análisis constituye el objeto del presente estudio.

Centrándonos en el examen de las razones que los niños dan para elegir o rechazar a sus compañeros, los estudios realizados ponen en evidencia que la aceptación está asociada con conductas de competencia social y con las habilidades necesarias para hacer y mantener relaciones satisfactorias entre iguales, como 
son conductas prosociales, habilidades comunicativas, de ayuda y cooperación y con ausencia de conductas agresivas (Fuentes, López, Eceiza y Aguirrezabala, 2001; Garaigordobil, 2005; Garaigordobil y García de Galdeano, 2006; Monjas, 2004). Por otra parte, se asume que el comportamiento agresivo es el principal correlato del rechazo de los iguales; aunque también se justifica por la conducta disruptiva o ansiosa (Coie y Koeppl, 1990; García-Bacete, Lara y Monjas, 2005; Leary, 2001; Monjas, Martín, García-Bacete y Sureda, 2005).

Williams y Asher (1993) en su trabajo "Las razones para el rechazo entre iguales" discuten por qué unos niños son aceptados y otros rechazados y comentan qué buscan los niños en su relación con los iguales. Señalan que cuando los niños deciden ser amigos de alguien, parece que se hacen a sí mismos unas preguntas esenciales: ¿Es divertido estar con este niño?, ¿nos influimos mutuamente en la dirección que a mí me gusta?, este niño, ¿es digno de confianza?, ¿me ayuda a conseguir mis metas?, ¿me hace sentir bien?, ¿es similar a mí? En función de las respuestas a estas seis preguntas, presentan una serie de características asociadas con la preferencia y con la exclusión (véase Tabla I). En síntesis, las siguientes son razones básicas de la aceptación: diversión, lealtad, influencia mutua, ayuda, contribución al propio bienestar y similaridad. Al contrario, en la base del rechazo están las conductas ser agresivo, dominante, disruptivo, traicionar la confianza, ser desconsiderado y ser diferente.

Ahondando más en las razones que justifican la preferencia de elección, la literatura ha subrayado los procesos que subyacen a los motivos de aceptación y/o rechazo. Al respecto, cabe distinguir dos grandes tradiciones: la primera afirma que los escolares efectúan su elección en función de un criterio dominante, esto es, la similaridad, la complementariedad o el bienestar. La segunda tradición apoyaría la tesis de que las preferencias o rechazos se realizan sobre la base del conjunto de comportamientos o características de los compañeros, recurriendo a una dinámica de análisis del coste-beneficio.

Los estudios de la primera tradición han señalado que infantes y adolescentes se eligen utilizando principalmente uno de los tres criterios siguientes. El primero se refiere a la similaridad o semejanzas entre el niño elector y el niño elegido, de forma que se tiende a evaluar positivamente lo que se nos parece y negativamente lo que vemos como diferente (Haselager, Hartup, van Lieshout y RiksenWalraven, 1998; Ladd, 2005; Morry, 2005). Preferimos a aquellos compañeros con los que compartimos algunas características, incluyendo las demográficas como el género o la edad, las biológicas como la maduración física y el atractivo facial y las conductuales como el logro académico, los intereses y actividades en común y la conducta agresiva (Gifford-Smith y Brownell, 2003). En este sentido no extraña la constatación de que durante toda la infancia se elija preferentemente a los del mismo género. García-Bacete (2006) confirma tanto el carácter intragénero de las nominaciones positivas como el sesgo hacia los chicos de las nominaciones negativas. El segundo criterio se centra en la complementariedad y diferencias y señala que en los amigos se buscan rasgos o intereses complementarios, lo que uno no tiene, lo diferente a lo propio; nos sentimos atraídos hacia aquellas características que no poseemos. El tercer criterio importante es el bienestar, la diversión y la satisfacción en la relación; simpatizamos con las personas que nos recompensan y nos aprecian y no estimamos y nos disgustan quiénes nos hacen pasarlo mal (Lafontana y Cillessen, 2002; Morry, 2005). Otros criterios señalados por la investigación y que pueden ser transversales a los señalados son la proximidad y la familiaridad, esto es, es más probable que se elija como amigos a los compañeros con los que se tiene relación, niños del entorno cercano, o conocidos con los que ya se han compartido actividades y espacios. 
TABLA I

Características asociadas con la aceptación y con el rechazo entre iguales

\begin{tabular}{|c|c|}
\hline ACEPTACIÓN & RECHAZO \\
\hline \multicolumn{2}{|c|}{ ¿Es divertido estar con este niño? } \\
\hline $\begin{array}{l}\text { - sentido del humor } \\
\text { - recursos, habilidad, competencia } \\
\text { - participación; se implica fácilmente } \\
\text { - cooperativo }\end{array}$ & $\begin{array}{l}\text { - agresivo, mezquino } \\
\text { - disruptivo } \\
\text { - mandón, dominante } \\
\text { - retraído, aprensivo o temeroso } \\
\text { - bajas habilidades cognitivas }\end{array}$ \\
\hline \multicolumn{2}{|c|}{ ¿Este niño es digno de confianza? } \\
\hline $\begin{array}{l}\text { - fiable } \\
\text { - honesto } \\
\text { - leal }\end{array}$ & $\begin{array}{l}\text { - agresivo, mezquino } \\
\text { - deshonesto } \\
\text { - traiciona la confianza }\end{array}$ \\
\hline \multicolumn{2}{|c|}{ ¿Nos influimos mutuamente en la dirección que a mí me gusta? } \\
\hline $\begin{array}{l}\text { - cooperativo } \\
\text { - sensible }\end{array}$ & $\begin{array}{l}\text { - agresivo, mezquino } \\
\text { - mandón, dominante } \\
\text { - inflexible, rígido }\end{array}$ \\
\hline \multicolumn{2}{|c|}{ ¿Este niño me ayuda a conseguir mis metas? } \\
\hline $\begin{array}{l}\text { - cooperativo } \\
\text { - servicial, atento, que ayuda }\end{array}$ & $\begin{array}{l}\text { - disruptivo } \\
\text { - impulsivo }\end{array}$ \\
\hline \multicolumn{2}{|c|}{ ¿Este niño me hace sentir bien conmigo mismo? } \\
\hline $\begin{array}{l}\text { - apoyo, amable } \\
\text { - sensible } \\
\text { - me gusta }\end{array}$ & $\begin{array}{l}\text { - insulta, descalifica } \\
\text { - no sensible } \\
\text { - me disgusta }\end{array}$ \\
\hline \multicolumn{2}{|c|}{ ¿Este niño es similar a mí? } \\
\hline $\begin{array}{l}\text { - intereses y valores comunes } \\
\text { - respeto a los usos y acuerdos de los iguales } \\
\text { - mismo género, raza y edad }\end{array}$ & $\begin{array}{l}\text { - diferentes intereses y valores } \\
\text { - no conformidad con los usos y } \\
\text { acuerdos de los iguales } \\
\text { - actitud y modos de superioridad } \\
\text { - discapacitado }\end{array}$ \\
\hline
\end{tabular}

Sin embargo, generalmente se alude a una mezcla entre semejanzas, diferencias y satisfacción. Ladd (2005) afirma que, como en la atracción interpersonal en la vida adulta, los niños se enrolan en interacciones de acuerdo con la "dinámica del coste-beneficio", es decir que maximizan los beneficios (diversión y satisfacción en el juego) y minimizan los costes (sufrimiento, aburrimiento o insatisfacción). La desproporción del coste frente al beneficio que las conductas agresivas conllevan, permite explicar el por qué los compañeros eventualmente excluyen a un colega de la actividad de grupo; por el contrario, si el sujeto presenta también una conducta prosocial y aporta al grupo más beneficios que costes (por ejemplo persona afectuosa, afirmativa, cooperativa...), se le acepta.

Inderbitzen (1991) estudió las conductas que son importantes para las relaciones positivas entre iguales en la adolescencia; para ello se aplicó a 1142 alumnos de 14-15 años una estrategia de nominación sociométrica y se les pidió que 
escribieran las razones para cada nominación. Concluyó que la relación positiva y la aceptación estaba asociada con cooperación, iniciación de actividades, disfrutar de chistes y bromas, compartir actividades e intereses en común, lealtad, amistad y apoyo. En la misma dirección Ladd (2005) indicó que una de las claves principales para establecer lazos de amistad con los iguales es desarrollar estrategias de colaboración, de comunicación y participar de intereses y actividades conjuntos que favorecen una mutua implicación emocional y reducen los conflictos que dicha relación pueda suponer.

Si nos ceñimos ahora a las razones que justifican el rechazo y la exclusión entre compañeros, hemos de remarcar que tanto niños como adolescentes señalan la agresión como la principal razón, llegando a indicarse de modo bastante unánime, la conexión entre agresión y rechazo. Sin embargo, cada vez de forma más contundente, se viene señalando que esto no es necesariamente así, sobre todo en la infancia tardía y en la adolescencia, etapa evolutiva en la que aparece relación entre conductas agresivas y buena aceptación-preferencia por los iguales (Bierman, Smoot y Aumiller, 1993). Por ejemplo Rodkin, Farmer, Peral y van Acker (2000) comentan que en las clases de secundaria aparecen dos tipos de alumnado popular y preferido: los populares-prosociales y competentes escolarmente y los populares-antisociales que son agresivos, disruptivos y no estudiosos. En la misma línea Luthar y McMahon (1996) y Kiesner y Pastore (2005) señalan que durante la adolescencia el comportamiento antisocial llega a asociarse positivamente con la popularidad entre los iguales. Opinan Williams y Asher (1993) que el que un niño tenga alguna característica negativa no quiere decir que será rechazado ya que la preferencia o el rechazo dependen del conjunto de sus comportamientos positivos y negativos, de suerte que las conductas positivas y atrayentes de un niño pueden esconder o minimizar algún comportamiento negativo; así un niño que a veces agrede puede ser aceptado por los demás porque es divertido, tiene buenas ideas y también ayuda.

\section{Objetivo}

Este estudio tiene tres objetivos: 1) Analizar los motivos por los que los niños y niñas de 10 y 11 años eligen y rechazan a sus iguales, articulándolos en un sistema de categorías, 2) Averiguar la frecuencia de los distintos motivos categorizados y 3) Establecer, de acuerdo con los resultados obtenidos, un catálogo de habilidades sociales para incorporar en los programas de intervención.

\section{Instrumento}

El instrumento utilizado para la recogida de datos es un cuestionario sociométrico de nominación entre iguales en el que se pide a cada alumno que haga tres nominaciones positivas y tres negativas de sus compañeros de acuerdo con el criterio de amistad (mejores amigos y menos amigos). Se pide además que razonen y justifiquen sus respuestas, y es precisamente este último aspecto lo que constituye el material básico de análisis para este estudio. Concretamente las preguntas son las siguientes:

1. ¿Quiénes son los tres compañeros y compañeras de esta clase que eliges como mejores amigos o amigas? ¿Por qué?

2. ¿Quiénes son los tres compañeros y compañeras de esta clase que menos te gustan como amigos o amigas? ¿Por qué?

\section{Participantes}

La muestra estuvo formada por 430 alumnos y alumnas de quinto y sexto curso de Educación Primaria, pertenecientes a 21 aulas de 8 centros públicos de 
Valladolid, Palma de Mallorca y Castellón de la Plana. De ellos 233 son varones $(52,5 \%)$ y 197 mujeres $(47,5 \%) ; 194$ son de $5^{\circ}$ curso $(43,8 \%)$ y 236 de $6^{\circ}$ curso (56,2\%); 78 participantes son de Valladolid (15,7\%), 91 de Palma de Mallorca $(16,1 \%)$ y 261 de Castellón de la Plana $(68,2 \%)$.

\section{Procedimiento}

El cuestionario sociométrico fue aplicado en cada aula por miembros del equipo de investigación. Después de unos previos contactos telefónicos y por escrito con los equipos directivos de los centros educativos a los que se le explicaban los objetivos del estudio, se concretaban los cursos, grupos y horario en los que se realizaría el cuestionario. El alumnado cumplimentó la prueba en una sesión de unos 30 minutos de duración. El profesorado recibió con posterioridad los resultados de su grupo-clase en forma de gráficos y sociograma.

\section{Proceso de categorización}

Para efectuar el análisis de las razones emitidas por cada alumno se ha adaptado el procedimiento basado en la teoría fundamentada "grouded theory" y expuesto por Carrero (1999) y que básicamente consiste en:

1. Recopilación literal de las razones que da cada alumno para justificar sus elecciones positivas y negativas.

2. Primera clasificación, que agrupa tipos de respuestas semejantes (evitando clasificaciones "a priori").

3. Inicio de un nuevo análisis de las categorías, comparando constantemente los tipos de respuestas y los agrupamientos generados (método comparativo constante). Las comparaciones son de tres tipos: respuesta/respuesta, respuesta/categoría y categoría/categoría. Este proceso de reclasificación continúa hasta que las nuevas respuestas no añaden ninguna información significativa a las categorías generadas.

4. Formulación de una definición de cada categoría, lo que provoca nuevos cambios.

\section{Resultados}

\section{Categorización de las razones de aceptación y rechazo}

Los participantes proporcionaron un total de 2803 respuestas como motivos de aceptación (una media de 6,52 razones por cada alumno emisor) y 1787 como razones del rechazo (una media de 4,16 razones por cada alumno emisor).

Como resultado del proceso de categorización efectuado a partir de las razones esgrimidas por el alumnado para justificar las preferencias y los rechazos, se obtuvieron nueve categorías de aceptación y quince de rechazo cuya descripción se presenta en las tablas II y III junto con expresiones literales de algunas de las respuestas aportadas por los niños.

\section{Motivos de preferencia y rechazos: porcentajes}

Los resultados indican que los niños de 10 y 11 años utilizan como justificación de sus preferencias, las siguientes razones (ver Tabla IV): En un primer bloque encontramos las características positivas de la otra persona, especialmente su simpatía y amabilidad (categoría 5), la diversión y el disfrute compartido (categoría 9) y la amistad (categoría 1). A continuación, encontramos un segundo bloque de motivos formado por las categorías "compañeros" (categoría 2) y "comunalidad" (categoría 8). Un tercer bloque está formado por las categorías 
TABLA II

Categorias y subcategorías de aceptación.

\begin{tabular}{|c|c|c|}
\hline $\begin{array}{l}\text { Categorías y } \\
\text { subcategorías de } \\
\text { aceptación }\end{array}$ & $\begin{array}{c}\text { Descripción } \\
\text { Respuestas que se refieren a... }\end{array}$ & Ejemplos \\
\hline $\begin{array}{l}\text { 1. Amistad } \\
\text { 1.1. Buenos amigos } \\
\text { 1.2. Características de amistad }\end{array}$ & $\begin{array}{l}\text { Ser buenos amigos y características de una } \\
\text { auténtica relación de amistad: confianza, } \\
\text { afectos, lealtad, comprensión, sentirse } \\
\text { escuchado, entenderse, ayuda incondicional, } \\
\text { apoyo emocional, disponibilidad de la otra } \\
\text { persona, consuelo, el "estar pendiente"... }\end{array}$ & $\begin{array}{l}\text { Es mi mejor amigola } \\
\text { Me comprende } \\
\text { Es muy cariñosola } \\
\text { Es sincero } \\
\text { Puedes confiar en él } \\
\text { Me bace mucho caso } \\
\text { Siempre se preocupa }\end{array}$ \\
\hline $\begin{array}{l}\text { 2. Compañerismo } \\
\text { 2.1. Buenos compañeros } \\
\text { 2.2. Características del } \\
\text { compañerismo }\end{array}$ & $\begin{array}{l}\text { Ser buenos compañeros y características y } \\
\text { conductas vinculadas con las relaciones entre } \\
\text { compañeros o colegas: ayuda, generosidad, } \\
\text { compañía, seguridad y protección }\end{array}$ & $\begin{array}{l}\text { Es una buena compañera } \\
\text { Me ayuda mucho } \\
\text { Comparte las cosas } \\
\text { Me hace compañia } \\
\text { Es solidario }\end{array}$ \\
\hline 3. Me cae bien & $\begin{array}{l}\text { Alusión de atracción, especificando } \\
\text { características del otro que le atraen y/o } \\
\text { señalan el gusto por estar juntos }\end{array}$ & $\begin{array}{l}\text { Me cae bien } \\
\text { Me gusta estar con ella } \\
\text { Me gusta su forma de ser }\end{array}$ \\
\hline 4. Características físicas & $\begin{array}{l}\text { Características externas: el atractivo, la } \\
\text { apariencia y el aspecto físico }\end{array}$ & $\begin{array}{l}\text { Es guapa } \\
\text { Es muy guapa }\end{array}$ \\
\hline 5. Simpatía & $\begin{array}{l}\text { Características y cualidades positivas del otro } \\
\text { niño, específicamente su simpatía y } \\
\text { amabilidad }\end{array}$ & $\begin{array}{l}\text { Es muy amable conmigo } \\
\text { Es majola } \\
\text { Es muy simpático }\end{array}$ \\
\hline $\begin{array}{l}\text { 6. Buen talante y } \\
\text { comportamiento } \\
\text { 6.1. Buen talante } \\
\text { 6.2. Ausencia de } \\
\text { conductas negativas }\end{array}$ & $\begin{array}{l}\text { La bondad de la persona en dos formas: } \\
\text { a) respuestas formuladas en positivo, que } \\
\text { incluyen juicios globales de la persona y de la } \\
\text { relación y b) respuestas formuladas en } \\
\text { negativo, indicando que el otro niño no } \\
\text { presenta conductas negativas que podrían } \\
\text { afectar a la relación }\end{array}$ & $\begin{array}{l}\text { Es buena persona } \\
\text { Tiene muy buen carácter } \\
\text { Nos llevamos bien } \\
\text { Nunca se enfada } \\
\text { No insulta } \\
\text { No se burla de los demás }\end{array}$ \\
\hline $\begin{array}{l}\text { 7. Competencia (escolar } \\
\text { y otras) }\end{array}$ & $\begin{array}{l}\text { Alusiones directas tanto a la inteligencia del } \\
\text { otro niño, a su capacidad para el estudio, a su } \\
\text { comportamiento en el centro escolar, a su } \\
\text { creatividad, etc., como a habilidades y } \\
\text { destrezas físicas y psicomotrices }\end{array}$ & $\begin{array}{l}\text { Es muy listo } \\
\text { Es muy estudiosa } \\
\text { Es guay } \\
\text { Es valiente } \\
\text { Es muy bueno en fútbol }\end{array}$ \\
\hline $\begin{array}{l}\text { 8. Comunalidad (en } \\
\text { común) } \\
\text { 8.1. Experiencias, gustos } \\
\text { y actividades en común } \\
\text { 8.2. Inicio de la relación } \\
\text { y red social común }\end{array}$ & $\begin{array}{l}\text { Aspectos comunes: a) tener experiencias en } \\
\text { común y pasar tiempo juntos y b) intereses y } \\
\text { gustos en común, c) relación que se inició } \\
\text { hace tiempo y d) red social común de } \\
\text { familiaridad y vecindad }\end{array}$ & $\begin{array}{l}\text { Hacemos los deberes juntas } \\
\text { Quedamos para salir } \\
\text { Nos gustan las mismas cosas } \\
\text { Es mi amigo desde que vine } \\
\text { a este colegio } \\
\text { Mis padres y los suyos son } \\
\text { amigos }\end{array}$ \\
\hline 9. Diversión & $\begin{array}{l}\text { Disfrute y satisfacción de la relación; } \\
\text { el otro niño es divertido, gracioso..., y el niño } \\
\text { emisor se divierte y se lo pasa bien con él }\end{array}$ & $\begin{array}{l}\text { Me río mucho con él } \\
\text { Es alegre } \\
\text { Hace muchas bromas } \\
\text { Cuando estoy con ella, no } \\
\text { me aburro }\end{array}$ \\
\hline
\end{tabular}


TABLA III

Categorías y subcategorías de rechazo

\begin{tabular}{|c|c|c|}
\hline $\begin{array}{l}\text { Categorías y } \\
\text { subcategorías de } \\
\text { rechazo }\end{array}$ & $\begin{array}{c}\text { Descripción } \\
\text { Respuestas que se refieren a... }\end{array}$ & Ejemplos \\
\hline $\begin{array}{l}\text { 1. Falta de amistad } \\
\text { 1.1. No ser buen amigo } \\
\text { 1.2. Ausencia de } \\
\text { características de } \\
\text { amistad } \\
\text { 1.2.1. Desleal } \\
\text { 1.2.2. Falta de reciprocidad }\end{array}$ & $\begin{array}{l}\text { No ser buenos amigos y características de una } \\
\text { mala relación de amistad como ser desleal y/o } \\
\text { mostrar una falta de reciprocidad hacia la otra } \\
\text { persona: chivarse, ser cotilla, entrometerse, no } \\
\text { escuchar, no hacer caso o no comprender... }\end{array}$ & $\begin{array}{l}\text { No es mi amigola } \\
\text { No se puede confiar en él } \\
\text { Es mentiroso } \\
\text { Es muy recelosola } \\
\text { Es injustola } \\
\text { No nos llevamos bien }\end{array}$ \\
\hline $\begin{array}{l}\text { 2. Mal compañero } \\
\text { 2.1. No es buen compañero } \\
\text { 2.2. Características de mal } \\
\text { compañero } \\
\text { 2.2.1. No ayuda } \\
\text { 2.2.2. Beneficio propio }\end{array}$ & $\begin{array}{l}\text { No ser buenos compañeros y características de } \\
\text { mal compañero, al no ayudar al otro y/o } \\
\text { buscar el beneficio propio: no comparte, se } \\
\text { aprovecha, es egoísta }\end{array}$ & $\begin{array}{l}\text { No te deja las cosas } \\
\text { Copia mis cosas } \\
\text { Es un interesadola } \\
\text { Tiene envidia }\end{array}$ \\
\hline 3. Me cae mal & $\begin{array}{l}\text { No agrado del otro, especificando } \\
\text { características que no gustan del otro niño }\end{array}$ & $\begin{array}{l}\text { No me gusta } \\
\text { No me agrada } \\
\text { Me cae mal }\end{array}$ \\
\hline 4. Características físicas & $\begin{array}{l}\text { Características externas del otro: apariencia } \\
\text { física desagradable y/o aspectos de higiene } \\
\text { poco cuidadosa }\end{array}$ & $\begin{array}{l}\text { Es feo } \\
\text { Es gordola } \\
\text { Huele mal } \\
\text { Se quita los mocos } \\
\text { No se peina }\end{array}$ \\
\hline 5. Antipatía & $\begin{array}{l}\text { Características y cualidades poco agradables } \\
\text { del otro niño, concretamente su antipatía }\end{array}$ & $\begin{array}{l}\text { Es muy antipáticola } \\
\text { No es una persona simpática } \\
\text { No es majola }\end{array}$ \\
\hline 6. Mal carácter & $\begin{array}{l}\text { Cualidades no positivas del otro niño: mal } \\
\text { humorado, negativo, que se enfada } \\
\text { constantemente, que protesta por todo y se } \\
\text { rebota por cualquier cosa }\end{array}$ & $\begin{array}{l}\text { Tiene un carácter muy fuerte } \\
\text { No acepta ninguna broma } \\
\text { Te contesta mal } \\
\text { Se pica por nada }\end{array}$ \\
\hline $\begin{array}{l}\text { 7. Mala competencia } \\
\text { académica }\end{array}$ & $\begin{array}{l}\text { Carencia de habilidades académicas y/o } \\
\text { comportamiento negativo en el centro escolar: } \\
\text { es vago, interrumpe, no viene a clase o se } \\
\text { porta mal, no hace los deberes, no trabaja }\end{array}$ & $\begin{array}{l}\text { No es buen/a estudiante } \\
\text { Es muy gandulla } \\
\text { No sabe nada } \\
\text { Es un analfabetola } \\
\text { Saca malas notas }\end{array}$ \\
\hline $\begin{array}{l}\text { 8. Aburrido/ Retraído } \\
\text { 8.1. Aburrido } \\
\text { 8.2. Retraído }\end{array}$ & $\begin{array}{l}\text { Relación poco atractiva, nada divertida; el otro } \\
\text { niño es soso, no tiene sentido del humor y con } \\
\text { él no se lo pasan bien. También se incluyen } \\
\text { las referidas a timidez, poca comunicación del } \\
\text { otro, calificándolo de persona rara y con la } \\
\text { que resulta difícil entenderse }\end{array}$ & $\begin{array}{l}\text { Nunca te cuenta chistes } \\
\text { No te ríes nada con élella } \\
\text { Es mustio } \\
\text { No es gracioso } \\
\text { No sabe hacer bromas } \\
\text { Nunca te babla } \\
\text { Es muy parada }\end{array}$ \\
\hline 9. Tonto-niñato, inmaduro & $\begin{array}{l}\text { Comportamiento infantil, indeciso; conductas } \\
\text { inmaduras, patosas, mimadas, queriendo } \\
\text { llamar la atención de los otros }\end{array}$ & $\begin{array}{l}\text { Se deja influir por otros } \\
\text { Es tontitola } \\
\text { Hace el bobo } \\
\text { Es imbécil } \\
\text { Es gallina }\end{array}$ \\
\hline
\end{tabular}


10. Pesado/ Molesto
Comportamiento molesto, agobiante, pesado y que no favorece una relación satisfactoria
Es un plasta, un pelma

No para de hablar

Es inaguantable

Me toca las narices

\section{Agresión verbal y} gestual

Me dice palabrotas

Se burla de mí
Conducta agresiva verbal y/o gestual manifestada en insultos, gestos feos y malas palabras
Habla mal de la gente

Se ríe de los demás

\begin{tabular}{|c|c|c|}
\hline $\begin{array}{l}\text { 12. Dominancia y } \\
\text { Superioridad } \\
\text { 12.1. Prepotencia } \\
\text { 12.2. Rechaza } \\
\text { 12.3. Manipula } \\
\text { 12.4. Intimida }\end{array}$ & $\begin{array}{l}\text { Dominancia o superioridad ejercida a través } \\
\text { de cuatro aspectos: a) prepotencia, chulería, } \\
\text { machismo, b) rechazo, no dejando jugar o } \\
\text { hablar a los otros, excluirlos; c) manipulación } \\
\text { del otro: meterlos en líos, inventarse cosas; d) } \\
\text { intimidación, tratando mal al otro, no } \\
\text { respetando, amenazando, despreciando, } \\
\text { faltando al respeto }\end{array}$ & $\begin{array}{l}\text { Se cree el rey del mundo } \\
\text { Es mandón } \\
\text { No deja jugar a los demás } \\
\text { No deja bablar a los demás } \\
\text { Habla mal de los otros } \\
\text { Es muy liante } \\
\text { Se pasa con los otros } \\
\text { Hace bromas pesadas }\end{array}$ \\
\hline 13. Agresión física & $\begin{array}{l}\text { Conductas de agresión física, lo que implica } \\
\text { una agresión activa y directa: pegar, arañar, } \\
\text { tirar del pelo, pegar bofetadas, empujar o } \\
\text { pellizcar }\end{array}$ & $\begin{array}{l}\text { Me empuja } \\
\text { Me pone zancadillas } \\
\text { Busca pelea } \\
\text { Quiere guerra }\end{array}$ \\
\hline 14. Antisocial/Vandalismo & $\begin{array}{l}\text { Conductas antisociales y que violan las } \\
\text { normas: maleducado, ladrón, tramposo }\end{array}$ & $\begin{array}{l}\text { Es gamberro } \\
\text { Destroza el material } \\
\text { Hace trampas }\end{array}$ \\
\hline 15. Falta de relación & $\begin{array}{l}\text { Ausencia de relación y de conocimiento del } \\
\text { otro, no se hablan, no juegan o no hacen cosas } \\
\text { juntos }\end{array}$ & $\begin{array}{l}\text { No viene conmigo } \\
\text { No la conozco bien } \\
\text { Siempre va con otros }\end{array}$ \\
\hline
\end{tabular}

"me cae bien", "buen talante y comportamiento" y "competencia”. La categoría "características físicas" sólo aporta un 1\% de las respuestas.

Los datos muestran que los niños eligen la dominancia-superioridad (categoría 12) como la razón más frecuente de rechazo hacia sus iguales, puesto que constituye el 21,4\% de las respuestas; de manera concreta el motivo "mostrar prepotencia" es el que obtiene porcentajes de respuesta más altos (un 14.8\%). A continuación, encontramos un bloque de motivos en torno al 10\% de las respuestas: agresión física (categoría 13), agresión verbal (categoría 11), tonto-niñato o inmaduro (categoría 9), me cae mal (categoría 3) y pesado-molesto (categoría 10). A continuación, en torno al $5 \%$ un nuevo bloque de motivos son: falta de amistad (categoría 1), aburrido/retraído (categoría 8) y no nos relacionamos (categoría 15). Con pocas respuestas aparecen las categorías: antisocial (categoría 14), antipático (categoría 5), mal compañero (categoría 2), mal estudiante (categoría 7), mal carácter (categoría 6). Finalmente, las características físicas (categoría 4) no son un motivo importante de rechazo en estas edades.

\section{Discusión y conclusiones}

Los resultados de este estudio ponen de relieve que las principales razones por las que se elige un amigo en el aula son la simpatía, la diversión, la satisfacción conjunta y la presencia de características relevantes en una relación de amistad (confianza, lealtad, apoyo, ayuda...). Por su parte, los principales motivos de rechazo esgrimidos se focalizan en las conductas agresivas; primeramente en la agresión psicológica e indirecta (dominancia y superioridad, especialmente pre- 
TABLA IV

Frecuencias totales de los motivos de aceptación

\begin{tabular}{lcc}
\hline \multicolumn{1}{c}{ Categorías } & \multicolumn{2}{c}{ Total } \\
\cline { 2 - 3 } & $\mathrm{N}$ & $\%$ \\
\hline 1.1. Buenos amigos & 242 & 8.7 \\
1.2. Características de amistad & 254 & 9.1 \\
1. Amistad & 496 & 17. \\
2.1. Buen compañero & 72 & 2.6 \\
2.2. Características del compañerismo & 251 & 9.0 \\
2. Compañerismo & 323 & 11.6 \\
3. Me cae bien & 234 & 8.4 \\
4. Características físicas & 27 & 1.0 \\
5. Simpatía & 549 & 19.7 \\
6.1. Buen talante & 118 & 4.2 \\
6.2. Ausencia de conductas negativas & 47 & 1.7 \\
6. Buen talante y comportamiento & 165 & 5.9 \\
7. Competencia & 110 & 4.0 \\
8.1. Experiencias, gustos y actividades en común & 231 & 8.3 \\
8.2. Inicio de la relación y red social común & 92 & 3.3 \\
8. Comunalidad & 323 & 11.6 \\
9. Diversión & 529 & 19.0 \\
Sin clasificar & 25 & 0.9 \\
\hline TOTAL & 2781 & 10.0 \\
\hline
\end{tabular}

potencia), después en la agresión física y finalmente en la agresión verbal. Los comportamientos que revelan inmadurez y falta de atención y los que agobian y/o resultan molestos para los demás son otras razones importantes de rechazo. Es preciso indicar que los motivos de rechazo en relación a cuestiones de apariencia física, mal carácter, aspectos académicos y conductas antisociales no obtienen porcentajes de rechazo elevados.

Estos resultados apoyan con la idea de que existen múltiples razones que conducen a la preferencia o al rechazo puesto que el alumnado de estas edades ha aportado un conjunto amplio de motivos A falta de análisis más específicos en los que se contraste los motivos emitidos y recibidos por cada alumno, estos resultados son congruentes con la evidencia disponible que afirma que los niños en esta edad no eligen a sus compañeros de juegos y actividades sólo por criterios de semejanza o diferencia, sino que muestran preferencias de relación debidas a muy diversas razones, en particular las que conlleva la búsqueda del bienestar. Los niños eligen como amigos a los que son simpáticos, agradables y majos, que son divertidos y graciosos y con los que se lo pasan bien y que además poseen conductas y habilidades involucradas en la amistad como entendimiento, ayuda, lealtad, confianza... Por supuesto que en muchos casos todo esto ocurre en el marco de actividades compartidas y tiempo pasado en común, lo que apoyaría la hipótesis de la semejanza de intereses, actividades y ocupaciones. Así pues, los resultados se enmarcarían más en la línea de la consideración de una multiplicidad de motivos, valorando su coste-beneficio (Bishop e Inderbitzen, 1995; Ladd, 2005; Morry, 2005).

Si analizamos los resultados obtenidos y comparamos las principales razones aportadas para justificar las preferencias de relación y elección con las que hacen lo propio respecto al desagrado en la interacción, comprobamos que, en su conjunto, existe un alto grado de paralelismo entre ambas taxonomías que constitu- 
TABLA V

Frecuencias totales de los motivos de rechazo

\begin{tabular}{|c|c|c|}
\hline \multirow[b]{2}{*}{ Categorías } & \multicolumn{2}{|c|}{ Total } \\
\hline & $\mathrm{N}$ & $\%$ \\
\hline 1.1.No ser buen amigo & 12 & 0.7 \\
\hline 1.2 Ausencia de características de amistad & 96 & 5.4 \\
\hline Desleal & 75 & 4.2 \\
\hline Falta de reciprocidad & 21 & 1.2 \\
\hline 1. Falta de amistad & 108 & 6.1 \\
\hline 2.1. No ser buen compañero & 2 & 0.1 \\
\hline 2.2 Característica de mal compañero/a & 61 & 3.5 \\
\hline No Ayuda & 36 & 2.0 \\
\hline Se aprovecha /beneficio propio & 25 & 1.4 \\
\hline 2. Mal compañero & 63 & 3.6 \\
\hline 3. Me cae mal, no me gusta & 150 & 8.5 \\
\hline 4. Características físicas & 14 & 0.8 \\
\hline 5. Antipatía & 63 & 3.6 \\
\hline 6. Mal carácter & 55 & 3.1 \\
\hline 7. Mala competencia académica & 56 & 3.2 \\
\hline 8.1 Aburrido & 49 & 2.8 \\
\hline 8.2. Retraído & 49 & 2.8 \\
\hline 8. Aburrido, retraído & 98 & 5.5 \\
\hline 9. Tonto-niñato o inmaduro & 153 & 8.7 \\
\hline 10. Pesado, molesto & 143 & 8.1 \\
\hline 11. Agresión verbal y gestual & 153 & 8.7 \\
\hline 12.1 Prepotencia & 261 & 14.8 \\
\hline 12.2 Rechaza & 28 & 1.6 \\
\hline 12.3 Manipula & 9 & 0.5 \\
\hline 12.4 Intimida & 77 & 4.4 \\
\hline 12. Dominancia/Superioridad & 379 & 21.4 \\
\hline 13. Agresión física & 186 & 11.5 \\
\hline 14. Antisocial/ Vandalismo & 65 & 3.7 \\
\hline 15. Falta de relación & 69 & 3.9 \\
\hline Sin clasificar & 17 & 1.0 \\
\hline TOTAL & 1768 & 100 \\
\hline
\end{tabular}

yen un afinado perfil de los aspectos involucrados en la competencia social y en el rechazo (Bishop e Inderbitzen, 1995; Garaigordobil y García de Galdeano, 2006; García-Bacete, 2007; Ladd, 2005; Merrell y Gimpel, 1998; Monjas, 2007). Considerados en su conjunto estos resultados están en consonancia con las características asociadas a la aceptación y el rechazo presentadas por Williams y Asher (1993) (ver Tabla VI).

Respecto de la conexión entre agresión y rechazo, nuestros resultados coinciden con los encontrados repetidamente en la literatura, los niños y adolescentes señalan la agresión como una razón para rechazar y excluir, o por lo menos para no estimar a un compañero (Coie, Dodge y Coppotelli, 1982; Leary, 2001; Merrell y Gimpell, 1998; Williams y Asher, 1993). Sin embargo, las cosas no son tan simples y a veces la agresión no conduce automáticamente al rechazo (Rodkin et al., 2000), ni el rechazo es consecuencia directa de la conducta agresiva. Entre los motivos de rechazo han surgido varias formas de agresión (física, dominancia, verbal y vandalismo). La forma de agresión que más se asocia con el rechazo en estas edades es la psicológica y la indirecta, es decir, la que se ejerce con intención de mostrar superioridad y dominar a los otros, mediante conduc- 
TABLA VI

Comparación con la Taxonomía de aceptación y rechazo entre iguales de Williams y Asher (1993).

\begin{tabular}{|c|c|c|}
\hline & ACEPTACIÓN & RECHAZO \\
\hline ¿Es divertido estar con este niño? & - Diversión & $\begin{array}{l}\text { - Aburrido/retraido } \\
\text { - Niñato } \\
\text { - Agresión Física }\end{array}$ \\
\hline ¿Este niño es digno de confianza? & - Amistad & $\begin{array}{l}\text { - Falta de Amistad } \\
\text { - Dominancia } \\
\text {-Agresión Física }\end{array}$ \\
\hline $\begin{array}{l}\text { ¿Nos influimos mutuamente en la dirección } \\
\text { que a mí me gusta? }\end{array}$ & $\begin{array}{l}\text { - Buen Talante } \\
\text { - Compañerismo }\end{array}$ & $\begin{array}{l}\text { - Mal carácter } \\
\text { - Dominancia } \\
\text {-Agresión Física } \\
\text { - Mal compañero }\end{array}$ \\
\hline ¿Este niño me ayuda a conseguir mis metas? & - Compañerismo & $\begin{array}{l}\text { - Mal Compañero } \\
\text { - Pesado } \\
\text { - Dominante }\end{array}$ \\
\hline $\begin{array}{l}\text { ¿Este niño me bace sentir bien conmigo } \\
\text { mismo? }\end{array}$ & $\begin{array}{l}\text { - Simpatía } \\
\text { - Me cae bien }\end{array}$ & $\begin{array}{l}\text { - Antipatía } \\
\text { - Me cae mal } \\
\text { - Agresión verbal }\end{array}$ \\
\hline ¿Este niño es similar a mí? & - Comunalidad & - Falta de relación \\
\hline
\end{tabular}

tas de prepotencia, exclusión, manipulación e intimidación. Esto nos lleva a enfatizar la relevancia que la agresividad psicológica tiene en la génesis del rechazo, aspecto que puede considerarse una contribución del presente estudio para la profundización en el análisis de las relaciones entre iguales puesto que muchos de los acercamientos anteriores se han centrado en la agresión física, verbal y gestual. Y es que precisamente conductas de dominancia, prepotencia o superioridad, suponen una alteración de lo que justamente caracteriza las relaciones entre iguales que por definición son paritarias y simétricas (Monjas, 2007; Ortega y del Rey, 2004; Trianes, 1996). Tiene además una importancia especial para el estudio de procesos de intimidación y acoso escolar (Díaz-Aguado, 2006; Monjas y Avilés, 2006).

Por otra parte, los resultados obtenidos en este estudio aportan elementos de ayuda para el diseño de un catálogo de habilidades sociales para incorporar en los programas de intervención. En concreto sería necesario incluir los siguientes aspectos: 1) utilizar modos cordiales de relación: simpatía, amabilidad..., 2) ser positivo, divertido y alegre: sentido del humor, emociones positivas..., 3) ser buen amigo: conductas de amistad (sinceridad, dar confianza, mostrar afecto...), prosociales (ayuda, consuelo, cooperación, preocupación por los demás) y de comunicación (saber escuchar...) y 4) utilizar estilo asertivo de relación, lo que implica desarrollar conductas igualitarias de relación y de respeto interpersonal y minimizar, tanto las conductas de agresividad, prepotencia y dominio, como las de inhibición, retraimiento y pasividad.

Las principales limitaciones de este estudio se derivan principalmente de la utilización de un único instrumento de evaluación en un diseño muy sencillo y con una muestra concreta. Todo ello obliga a ser cautelosos y moderados en las afirmaciones de nuestros hallazgos que, también han de limitarse estrictamente a la franja de edad con la que estamos trabajando, 10 y 11 años. Esto nos lleva a señalar que las futuras investigaciones han de utilizar distintas fuentes y diversos 
instrumentos de evaluación de la competencia social, han de aumentar la muestra y ampliar el rango de edad de los participantes.

Para finalizar hemos de dejar constancia de que este estudio tiene implicaciones educativas, puesto que si tenemos en cuenta los aspectos involucrados en la aceptación y el rechazo interpersonal, podremos provocar intencionalmente su promoción y desarrollo y/o su minimización a través de Programas de competencia social. En síntesis, conductas como las apuntadas en el catálogo, como son amabilidad, simpatía, disfrute conjunto, reírse y hacer bromas, mostrar afecto, apoyo emocional, comprensión, ayuda, consuelo, ser leal y confiable, respetar a los demás y establecer relaciones igualitarias de camaradería y amistad, mostrando un estilo de relación asertivo, parecen ser ciertamente relevantes como también han señalado distintos programas existentes (Luca de Tena, Rodríguez y Sureda, 2004; Merrell y Gimpel, 1998; Monjas, 2004; 2007; Ortega y del Rey, 2004), por lo que se considera necesario incluirlas en el trabajo sistemático para mejorar la calidad de las relaciones de convivencia en el contexto escolar.

\section{Referencias}

BiERMAN, K., SMOOT, D. \& AUMILLER, K. (1993). Characteristics of aggressive-rejected, aggressive (nonrejected), and rejected (nonaggressive) boys. Child Development, 64, 139-151.

BisHOP, J. \& INDERBITZEN, H. M. (1995). Peer acceptance and friendship: An investigation. Journal of Early Adolescence, 15 (4), 476-489.

BukOWSKI, A. F., NewCOMB, A. F. \& HARTup, W. W. (Eds.) (1998). The company they keep: Friendships in childhood and adolescence. Nueva York, NY: Cambridge University.

BukOwSKI, W. M., SiPPOLA, L., HoZA \& NewCOMB, A. F. (2000). Pages from a sociometric notebook: An analysis of nomination and rating scale measures of acceptance, rejection and social preference. New Directions for Child and Adolescent Development, 88, 11-26.

CARrero, V. (1999). Análisis Cualitativo de Datos: Aplicación de la Teoría de la Fundamentación (grounded theory) en el ámbito de la innovación organizacional. Tesis Doctoral. Castellón: Universitat Jaume I.

Cava, M. J. \& Musitu, G. (2001). Autoestima y percepción del clima escolar en niños con problemas de integración social en el aula. Revista de Psicología General y Aplicada, 54 (2), 297-311.

Coie, J. D., DODGe, K. A. \& Coppotelli, H. (1982). Dimensions and types of social status: A cross-age perspective. Developmental Psychology, 18 (4), 557-570.

COIE, J. D. \& KoEPPL, G. K. (1990). Adapting intervention to the problems of aggressive and disruptive rejected children. En S. R. Asher \& J. D. Coie (Eds.), Peer rejection in childhood (pp 309-337). Cambridge, UK: Cambridge University Press.

DíAz-AguAdo, M. J. (2006). Del acoso escolar a la cooperación en las aulas. Madrid: Pearson Educación.

Foster, S. L., INDERBITZEN, H. M. \& NAGLE, D. W. (1999). Assessing acceptance and social skills with peers in childhood. Behavior Modification, 17, 255-286.

Fuentes, M. J., LÓPEZ, F., EceizA, A. \& Aguirrezabala, E. (2001). Predictores emocionales y conductuales de la aceptación de los compañeros en la edad escolar. Revista de Psicología Social, 16 (3), 275-291.

GARAIGORDOBIL, M. (2005). Conducta antisocial durante la adolescencia: Correlatos socio-emocionales, predictores y diferencias de género. Psicología Conductual, 13 (2), 197-215.

Garaigordobil, M. \& GARCía de GaLDEANO, P. (2006). Empatía en niños de 10 a 12 años. Psicothema, 18 (2), 180-186.

GARCíA-BACETE, F. J. (2006). La identificación de los alumnos rechazados. Comparación de métodos sociométricos de nominaciones bidimensionales. Infancia y Aprendizaje, 29 (4), 437-451.

GARCíA-BACETE, F. J. (2007). La identificación de alumnos rechazados, preferidos, ignorados y controvertidos en el aula. Revista de Psicología General y Aplicada, 60 (1-2), 25-46.

García-BACete, F. J., LARA, A. \& MONJAS, I. (2005). ¿Por qué los niños no quieren jugar con otros niños? Un análisis exploratorio de los motivos de rechazo entre iguales. En I. Fajardo, F. Vicente, A. Ventura, I, Ruíz \& J. A. del Barrio (Eds.), Aportaciones psicológicas y mundo actual dando respuestas (pp. 257-268). Badajoz: Psicoex.

GarCía-BACETE, F. J., SUREDA, I. \& MONJAS, M. I. (2008). Distribución sociométrica en las aulas de chicas y chicos a lo largo de la escolaridad. Revista de Psicología Social, 23 (1), 63-74.

GifFord-Smith, M. E. \& BRownELL, C. A. (2003). Childhood peer relationships: social acceptance, friendships, and peer networks. Journal of School Psychology, 41, 235-284.

Haselager, G. J., Hartup, W. W., van Lieshout, C. F. M. \& Riksen-Walraven, J. M. A. (1998). Similarities between friends and nonfriends in middel childhood. Child Development, 69, 1198-1208.

INDERBITZEN, H. M. (1991). Identification of social behaviors important for adolescent peer acceptance: Implications for social skills training. Annual Meeting of the Association for Advancement of Behavior Therapy. Nueva York, 21-24 Noviembre.

KIESNER, J. \& PASTORE, M. (2005). Differences in the relations between antisocial behavior and peer acceptance across contexts and across adolescence. Child Development, 76 (6), 1278-93.

LADD, G. W. (2005). Children's peer relations and social competence: a century of progress. Yale: Yale University.

LAFontANA, K. M. \& Cillessen, A. H. (2002). Children's perceptions of popular and unpopular peers: A multimethod assessment. Developmental Psychology, 38 (5), 635-647.

LEARY, M. (2001). Interpersonal rejection. Nueva York: Oxford University Press.

LuCa De Tena, C., RodRíguez, R. I. \& SuREDA, I. (2004). Programa de Habilidades Sociales en la Enseñanza Secundaria Obligatoria. Málaga: Aljibe. 
492 Cultura y Educación, 2008, 20 (4), pp. 479-492

LutHAR, S. \& MCMAHON, T. (1996). Peer reputation among inner city adolescents: Structure and correlates. Journal of Research on Adolescence, 6, 581-603.

Merrell, K. W. \& Gimpel, G. A. (1998). Social skills of children and adolescents: Conceptualization, assessment, treatment. Mahwa, NJ: Lawrence Earlbaum Associates.

MONJAS, M. ${ }^{a}$ I \& Avilés, J. M. ${ }^{a}$ (2006). Programa de sensibilización contra el maltrato entre iguales. Colegas, amig@s y compañer@s. La familia ante el maltrato entre iguales. $2^{a}$ edición. Valladolid: Junta de Castilla y León y REA.

MONJAS, M. I. (2004). Programa de enseñanza de habilidades de interacción social (PEHIS) para niños y adolescentes. Madrid: CEPE. $\left(1^{\mathrm{a}}\right.$ ed., $7^{\mathrm{a}}$ reimp.).

MONJAS, M. I. (Dir.) (2007). Cómo promover la convivencia: Programa de asertividad y habilidades sociales (PAHS). Madrid: CEPE.

Monjas, M. I., Martín, I., García-BACETE, F. \& SuREdA, I. (2005). Razones que justifican la aceptación y el rechazo entre iguales. En I. Fajardo, F. Vicente, A. Ventura, I. Ruiz \& J. A. del Barrio. Aportaciones psicológicas y mundo actual. Dando respuestas (pp. 395-400). Badajoz: Psicoex.

Morry, M. M. (2005). Relationship satisfaction as a predictor of similarity ratings: A test of the attaction-similarity hypothesis. Journal of Social and Personal Relationships, 22 (4), 561-584.

Newcomb, A. F., Bukowski, W. M. \& Pattee, L. (1993). Children's peer relations: A meta-analytic review of popular, rejected, neglected, and average sociometric status. Psychological Bulletin, 113 (1), 99-128.

ORTEGA, R. \& DEL ReY, R. (2004). Construir la convivencia. Barcelona: Edebé.

Rodkin, P. C., FARmer, T. W., Pearl, R. \& VAN ACKer, R. (2000). Heterogeneity of popular boys: antisocial and prosocial configurations. Developmental Psychology, 36, 14-24.

TRIANES, M. V. (1996). Educación y competencia social. Un programa en el aula. Málaga: Aljibe.

Williams, G. \& Asher, S. R. (1993). Children without friends, Part 2: The reasons for peer rejection. En C. M. Todd (Ed), Day care center connections (pp. 3-5). Urbana-Champaign, IL: Univestity of Illinois Cooperative Extension Service. National Network for Child Care: http://www.nncc.org/Guidance/dc31_wo.friends2.html 